\title{
La gestion des ressources en eaux souterraines en région parisienne ouest
}

\author{
Michel Detay (*), Diane d'Arras (*), Pierre Suzanne (**)
}

\section{Préambule}

La Lyonnaise des Eaux a été créée en 1880 pour assurer la conception, le financement, la réalisation et la gestion des services publics d'eau, de gaz, et d'électricité. Après s'être recentrée en 1946 sur l'alimentation en eau potable, l'entreprise se diversifie à partir des années 1970 pour répondre à l'évolution des besoins et des attentes des collectivités. Elle a fusionné avec Dumez en 1990 pour former la Lyonnaise des Eaux-Dumez (LED), grand groupe de services, pour l'aménagement du cadre de vie et la gestion de l'environnement. Il regroupe 110000 collaborateurs et réalise un chiffre d'affaires supérieur à 80 milliards de francs.

La Direction Régionale Parisienne Ouest (RPO) est l'une des 14 Directions Régionales de la LED. Elle a vocation de gérer les services d'eau et d'assainissement dans l'Ouest de Paris: Hauts-de-Seine, Yvelines, Vald'Oise et Eure-et-Loire. Elle contribue directement ou indirectement à l'alimentation en eau d'un million de personnes. Pour ce faire elle produit chaque année 110 millions de mètres cubes $\left(\mathrm{Mm}^{3}\right)$ d'eau potable. La totalité des eaux distribuées provient de nappes souterraines.

\begin{abstract}
Dans cette note les auteurs dégagent les grandes idées et les différentes étapes qui ont prévalu à l'exploitation optimale des eaux souterraines et à la gestion du risque de pollution en région parisienne Ouest. Il s'agit plus ici de dégager les idées directrices et les choix stratégiques que d'expliquer dans le détail telle ou telle méthode.

Les grands centres de production de RPO sont répertoriés dans le tableau 1, page suivante.

La capacité des usines de traitement de réalimentation de nappe est respectivement de $30000 \mathrm{~m}^{3} \cdot \mathrm{j}^{-1}$ pour Aubergenville et de $150000 \mathrm{~m}^{3} \cdot \mathrm{j}^{-1}$ pour le Pecq-Croissy.

Nous présentons les principaux résultats acquis dans les champs captants d'Aubergenville et du Pecq-Croissy (fig. 1). Enfin nous exposons les outils de la sécurité mis en place pour faire face au risque de pollution accidentelle.
\end{abstract}

(*) Lyonnaise des Eaux-Dumez, Direction Régionale Parisienne Ouest, 42, rue du Président Wilson, BP 56, 78230 Le Pecq, France.

(**) Lyonnaise des Eaux-Dumez, Direction Technique de la Recherche, 72, rue de la Liberté, 92022 Nanterre Cedex, France.

\section{Groundwater resources management in the West of Paris}

The growth of the Parisian urban center has set the problem of potable water for more than a century. During the last decade the main concern of water distributors has been focused on water quantity and quality. Through two concrete examples the authors analyze the problematic, the methodology and results obtained for groundwater resource management in the West Paris area. They explain on site procedures, experimentations carried out and the realized numerical approach. A statement of the various surveys is proposed, which gives the main axes of future programs : hydrogeochemical survey, groundwater modeling, aquifer recharge optimization, water quality monitoring station, maintenance services. Finally authors explain the water pollution management strategy as well as the process used to preserve groundwater quality and quantity in case of major pollution. 


\section{Tableau 1. - Principales caractéristiques des centres de productions de la LED} en Région Parisienne Ouest.

\begin{tabular}{lcc}
\hline \multicolumn{1}{c}{ Centre de Production } & Aquifère & Capacité en $\mathrm{m}^{3} \cdot \mathrm{j}^{-1}$ \\
\hline Usine d'Aubergenville (78) & Craie & 150000 \\
Usine du Pecq-Croissy (78) & Craie & 180000 \\
Usine de Croissy rue des Ponts (78) & Craie & 4500 \\
Usine de St Germain en Laye (78) & Craie & 12000 \\
Forage de St Germain en Laye (78) & Albien & 3000 \\
Usine de Poissy-Migneaux (78) & Albien & 4500 \\
Usine de Verneuil-Vernouillet (78) & Alluvions & 11000 \\
Usine de la Chapelle (78) & Craie & 15000 \\
Captage du Pontel (78) & Calcaires grossiers & 3500 \\
Captage de la Falaise Nezel (78) & Craie & 3500 \\
Captage d'Aulnay-Maule (78) & Craie & 4000 \\
Captage de Mantes la Ville (78) & Craie & 9000 \\
Usine de Rueil Malmaison (92) & Craie & 30000 \\
Usine de Villeneuve la Garenne (92) & Albien/Sparnacien & 40000 \\
Nanterre & Albien & 5000 \\
\hline
\end{tabular}

1.

STATION O'Ooseravation de La
OUALITE OE LEAU QUALITE OE L'EAU
WATER OUALITY MONITORING STATION

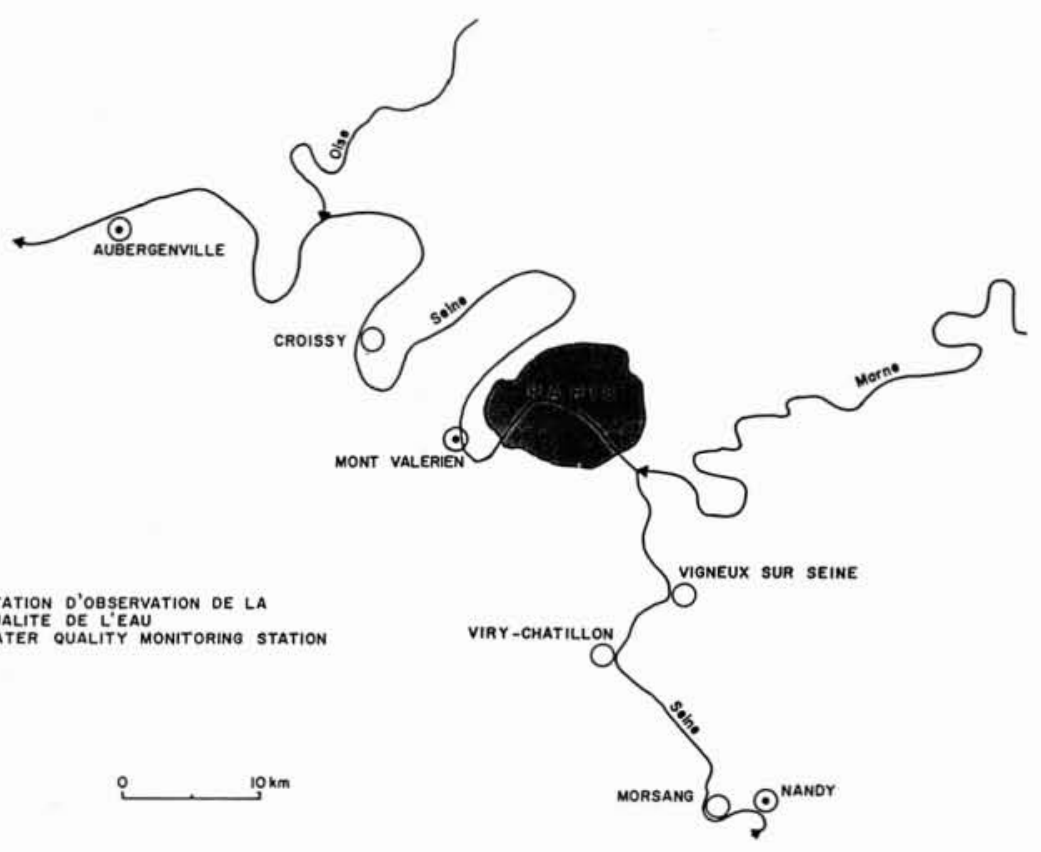

\section{Le site d'Aubergenville}

En zone Seine-aval, à Aubergenville, la LED exploite en rive gauche de la Seine, depuis plus de 20 ans, un ensemble de forages qui captent l'aquifère de la craie sénonienne. La nappe phréatique circule dans un ensemble craie-alluvions. Elle est en communication hydraulique avec le fleuve. Les prélèvements de la LED sont de l'ordre de $45 \mathrm{Mm}^{3} \cdot \mathrm{an}^{-1}$. L'aquifère est également exploité par des industriels, principalement par la Régie Renault à raison de 5 à $6 \mathrm{Mm}^{3}$.an-1.

Dès 1976, la LED a entrepris, avec l'aide de l'Agence Financière de Bassin Seine Normandie (AFBSN), une série d'études visant à appréhender les mécanismes hydrogéologiques mis en jeu et à quantifier le risque de pollution. Pour répondre à cette problématique plusieurs modèles mathématiques de l'aquifère ont été réalisés. Une fois le modèle hydrodynamique calé en régimes permanent et transitoire, une étude des risques de pollution a été entreprise, puis un modèle hydrodispersif a été élaboré. Ces études sont le fruit des travaux entrepris de 1984 à 1986 par les Sociétés ARLAB, SAFEGE et CPGF. Elles ont été financées par l'AFBSN, la LED et réalisées avec l'appui de la Direction de l'industrie de la recherche et de l'environnement (DRIRE). 
2. Aubergenville. Calage du modèle mathématique.

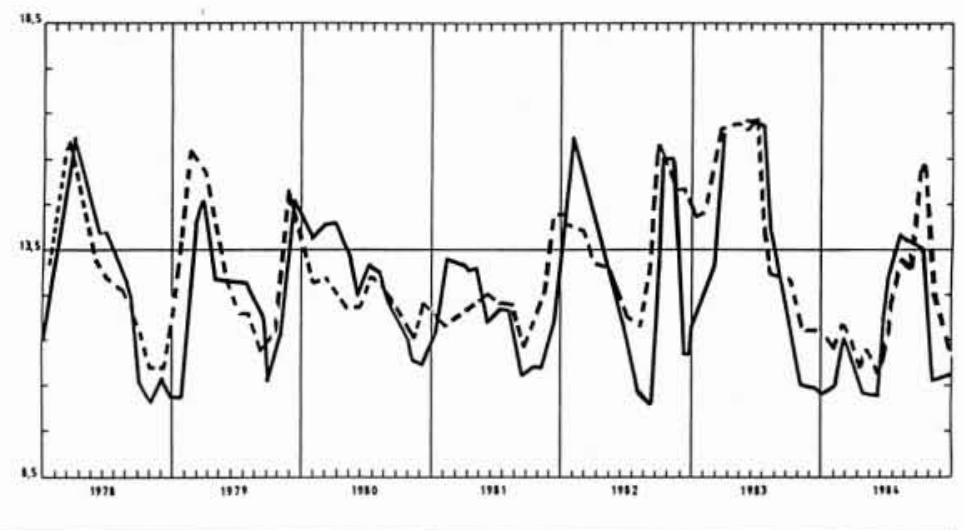

\subsection{Cadre hydrogéologique et mécanismes mis en jeu}

Les formations aquifères sont au nombre de deux :

- la craie sénonienne, très perméable dans les vallées et dans les parties supérieures de la formation. Elle devient compacte et très peu perméable à partir de $30-40 \mathrm{~m}$ de profondeur et sous recouvrement épais. De part et d'autre de la vallée de la Seine, la craie devient un aquiclude, se trouvant sous couverture tertiaire (Montien, Sparnacien);

- les alluvions, anciennes et récentes, déposées par la Seine (sables et graviers) qui recouvrent la craie sur une épaisseur de 10 à $20 \mathrm{~m}$.

Au niveau des mécanismes hydrogéologiques mis en jeu on distingue les entrées :

- apports de la Seine, à travers les dépôts du fond du lit du fleuve, provoquant de fortes pertes de charge entre le niveau de la Seine et les niveaux piézométriques du champ captant ;

- les apports latéraux sur les flancs des vallées;

- les apports dus à l'infiltration de la pluie sur l'aquifère ;

- les apports éventuels en amont et en aval du domaine d'étude ;

- la recharge artificielle de la nappe,

et les sorties :

- les écoulements souterrains à l'aval du système aquifère étudié ;

- les prélèvements par forages.

\subsection{Les modèles hydrodynamiques}

\subsubsection{Le premier modele}

Réalisé en régime permanent en 1976, ses objectifs étaient les suivants :

- préciser l'importance relative des différents flux et de fournir un bilan ;

— effectuer des tests de sensibilité sur les principaux paramètres introduits ;

- réaliser des simulations exploratoires (techniques des scenarii),

Le modèle comportait $\mathbf{4 7 5}$ mailles de taille variable comprise entre 2500 et $635 \mathrm{~m}$ de côté. Après contrôle de son calage, la zone des captages a été rediscrétisée avec des mailles plus petites de $300 \mathrm{~m}$ de côté pour obtenir une meilleure précision dans les calculs. La Seine a été, pour sa part, modélisée sur une seconde couche séparée de la couche principale par un semi perméable simulant le colmatage du lit du cours d'eau. Les simulations de gestion ont été effectuées en régime permanent autour d'une situation moyenne de la piézométrie.

Les points forts de ce travail ont été le calage des paramètres, la compréhension des mécanismes d'alimentation par la Seine et par les coteaux, l'analyse de l'efficacité de la réalimentation artificielle. Il est notamment apparu que :

- les transmissivités ayant permis de reproduire au mieux la piézométrie sur l'ensemble du domaine sont comprises entre 1 et $40.10^{-3} \mathrm{~m}^{2} \cdot \mathrm{s}^{-1}$ dans les vallées, et entre 0,1 et $1 \cdot 10^{-3} \mathrm{~m}^{2} \cdot \mathrm{s}^{-1}$ lorsque la craie est sous couverture ;

- la Seine apporte entre 60 et $70 \%$ de l'alimentation de la nappe ;

- les autres apports sont constitués par l'infiltration efficace $\left(I_{\text {eff }}\right.$ sur la vallée alluviale à raison de $25 \%$ de la pluie moyenne $(600 \mathrm{~mm})$, et sur les coteaux à raison de $5 \%$ de la pluie. Des tests de sensibilité montrant le rôle majeur de l' $I_{\text {eff }}$ sur la vallée de la Seine et les répercussions des années sèches sur l'aquifère.

Une série de scenarii a par ailleurs permis de localiser le meilleur site de réalimentaton artificielle de la nappe du point de vue hydraulique, c'est-à-dire celui qui diminue le plus les effets de pompage au plan de la piézométrie et des échanges nappe-rivière. Dès 1978 , le site retenu sur le modèle a été aménagé, et la réalimentation artificielle à partir d'eau de Seine traitée permet aujourd'hui d'exploiter l'aquifère au mieux de ses possibilités.

\subsubsection{Le modèle hydrodynamique en régime transitoire}

La perspective de la mise en œuvre d'un modèle hydrodispersif avec des mailles adaptées au problème physique a nécessité de discrétiser l'aquifère avec des mailles de $250 \mathrm{~m}$ de côté, ce qui nous a amené à un modèle comprenant 11600 mailles.

L'étalonnage de ce nouveau modèle hydraulique a été effectué sur une période de sept ans. L'ensemble des informations relatives aux prélèvements par pompage, à la réalimentation de la nappe, à la pluviométrie, aux niveaux de la Seine, à la piézométrie de la nappe a été saisi mensuellement sur ordinateur en vue de leur traitement sur le modèle. La validité de cet étalonnage se matérialise par la bonne coïncidence entre la piézométrie mesurée et calculée sur les 7 années de simulation (fig. 2). 


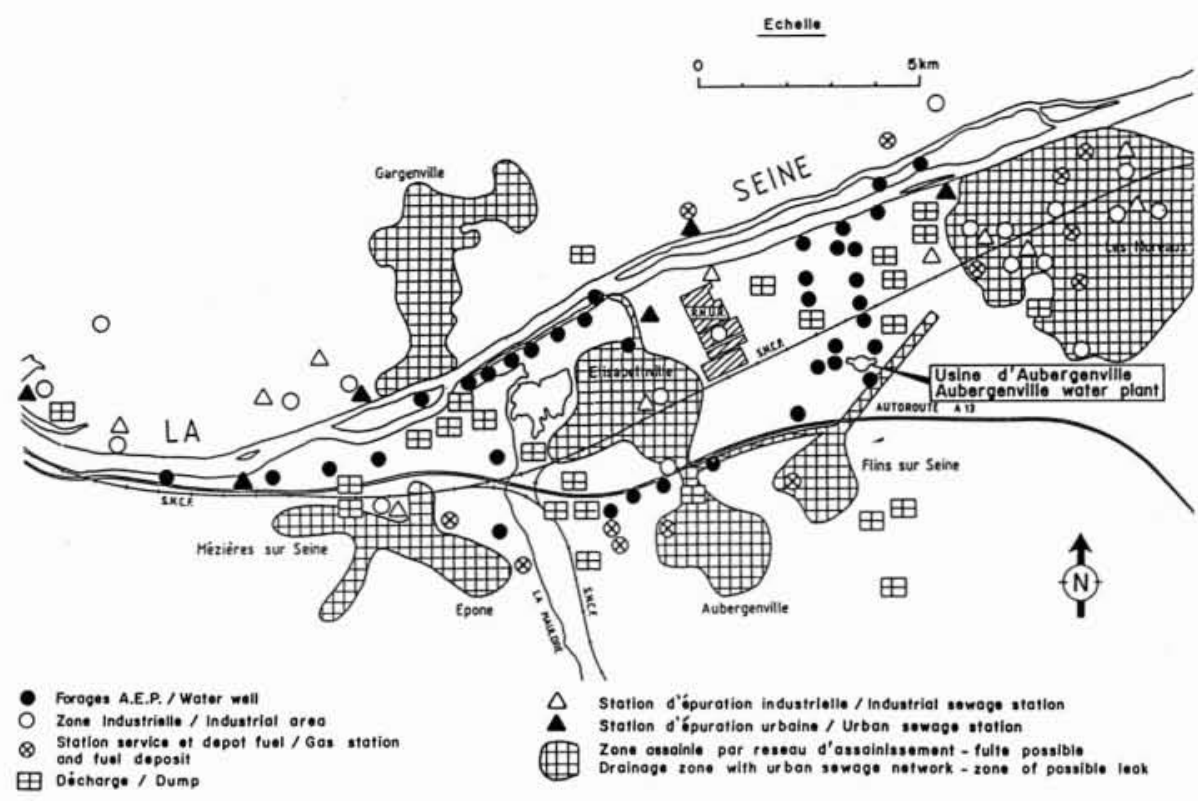

3. Aubergenville. Carte des risques potentiels de pollution.

\subsection{Les études de vulnérabilité de la nappe}

En 1984-1985 l'étude de vulnérabilité de la nappe d'Aubergenville a été réalisée. Elle s'inscrit dans le cadre des actions entreprises au titre de la sécurité de l'alimentation en eau potable de la Région Parisienne. Le but de l'étude était de répondre aux interrogations suivantes :

- Quel est l'état actuel de la nappe ?

- Une pollution accidentelle due aux infrastructures, hors installations industrielles, peut-elle avoir lieu ?

$\mathrm{Si}$ oui, dans quelle zone et par quels produits?

- Quels seront les aménagements éventuels du site à prévoir pour en diminuer l'incidence ?

L'étude a comporté plusieurs phases: recensement des problèmes et recensement des sources potentielles de pollution hors activité industrielle. L'évaluation des risques de pollution dus aux établissements industriels a été réalisée par la DRIRE. Une carte synthétique présentant les principaux risques de pollution du site a été élaborée (fig. 3).

Parallèlement une étude a été confiée à CPGF pour évaluer les vitesses d'écoulement et les propriétés dispersives de la nappe. Ce travail a comporté trois opérations :

1. Une observation semi-quantitative des variations naturelles des concentrations en tritium de la nappe et de la Seine. Le tritium a été choisi dans la mesure où il s'agit d'un traceur parfait de l'eau, faisant partie intégrante de la molécule d'eau. De plus, des études précédentes avaient montré qu'il existait un contraste suffisant entre les concentrations en tritium des différentes sources d'alimentation potentielle : Seine, nappe locale (alimentée par les précipitations), craie.

Les concentrations type des trois sources potentielles sont les suivantes :

Pôle craie : ${ }^{3} \mathrm{H}=5$ UT.

Pôle Seine : ${ }^{3} \mathrm{H}=50 \pm 2 \mathrm{UT}$.

Pôle nappe : ${ }^{3} \mathrm{H}=64 \pm 2$ UT

Cette étude confirme les résultats acquis par le modèle, notamment que $70 \%$ de l'eau pompée provient de la Seine.
2. Un traçage par de l'iodure de sodium, traceur classique, conforté par un traçage au chlorure de sodium. Les conclusions essentielles de ce travail montrent que :

- la porosité est comprise entre 0,3 et $3 \%$;

- les vitesses sont de l'ordre de $12 \mathrm{~m} \cdot \mathrm{h}^{-1}$;

- les coefficients de dispersion intrinsèque sont de l'ordre de 10 à $15 \mathrm{~m}$ pour les parcours de l'ordre de $100 \mathrm{~m}, 0,5$ à $1 \mathrm{~m}$ pour les parcours de 10 à $15 \mathrm{~m}$.

3. Des traçages globaux par injection en bord de Seine de $\mathrm{NaCl}$, en contrôlant sa progression par des mesures de résistivité du sol effectuées par prospection géoélectrique. On constate une vitesse apparente de l'ordre de $15 \mathrm{~m} \cdot \mathrm{h}^{-1}$.

Cette phase d'étude a permis d'évaluer, à la suite de 9 essais en régime radial convergent et de deux essais globaux, les valeurs de porosité cinématique $\left(\omega_{c}\right)$ et du coefficient de dispersion longitudinale $\left(D_{L}\right)$ :

$-\omega_{c}=0,5$ à $3,5 \%$;

$-D_{L}=0,5$ à $1,5 \mathrm{~m}$ pour les transferts « courts " (10 à $100 \mathrm{~m}), 10$ à $15 \mathrm{~m}$ pour les transferts «longs » $(>100 \mathrm{~m})$. Compte tenu des résultats des essais globaux, on retiendra plutôt des valeurs hautes de $D_{L}$, soit 1,5 et $15 \mathrm{~m}$.

Enfin, l'étude semi-quantitative a, par ailleurs, permis d'identifier pour chaque ouvrage les parts respectives d'eau de Seine et d'eau de nappe dans leur alimentation [CPGF, 1985]. Cette approche permet de préciser la vulnérabilité de chacun des ouvrages vis-à-vis d'une contamination, soit de la Seine, soit de la nappe.

\subsection{Le modèle hydrodispersif}

En 1985/1986, une seconde étude a été engagée au moyen d'un modèle hydrodispersif dans le but d'évaluer les risques de pollution accidentelle par la Seine ou par un accident ponctuel survenant dans le voisinage des captages, et les moyens à mettre en œuvre pour évacuer cette pollution. Les objectifs étaient : 
— d'affiner le modèle de 1976 et de passer en régime transitoire ;

- la compréhension des mécanismes d'alimentation par la Seine et les coteaux ;

— l'analyse de l'efficacité de la réalimentation artificielle ;

- d'étudier les propriétés hydrodispersives de la nappe ;

- de modéliser les phénomènes de dispersion ;

- d'évaluer la vulnérabilité de la nappe et de définir une "politique de crise" en cas de pollution.

Le modèle hydrodispersif a été construit autour de plusieurs idées, exposées ci-après.

\subsubsection{Physique du modèle}

En premier lieu, la propagation des polluants se fait le long des filets de courants, et il s'ajoute une dispersion à l'écoulement proprement dit. Cette dispersion est plus forte le long des filets de courant que transversalement. La dispersion est donc caractérisée par deux coefficients, un coefficient de dispersion longitudinale, et un coefficient de dispersion transversale. Ces coefficients sont par ailleurs proportionnels à la vitesse du fluide.

En schématisant, on peut dire que les équations de la dispersion montrent que le phénomène est équivalent à un transport le long des lignes de courant, à la vitesse du fluide, et, simultanemént, à une dispersion qui est tout à fait analogue à ce que l'on observe en hydraulique souterraine lorsque le cône d'influence d'un pompage s'élargit.

L'équation générale s'écrit :

$\operatorname{div}(b \cdot \overline{\bar{D}} \cdot \overrightarrow{\operatorname{grad}} C)+\psi \cdot \frac{C_{p}}{u}=\frac{\delta(C \cdot b)}{\delta t}-\operatorname{div}(b \cdot C \cdot \bar{V})$

avec :

$b$ épaisseur du terrain saturé

$\overline{\bar{D}}$ tenseur des dispersions

$\Psi$ flux d'eau polluée injectée par accident

$C_{p}$ concentration du flux polluant

$\bar{V}$ vitesse de l'eau dans le sol

$u$ porosité du sol.

Par ailleurs, les coefficients de dispersion $D_{L}$ et $D_{T}$ longitudinale et transversale sont proportionnels à la vitesse effective du fluide. La dispersion n'est pas un phénomène isotrope : le fluide disperse plus dans le sens du courant que dans le sens transversal.

En pratique, si l'on appelle la dispersion longitudinale $\left(D_{L}\right)$ et transversale $\left(D_{T}\right)$, et $\bar{V}$ la vitesse du fluide :

$$
\begin{aligned}
& D_{L}=\alpha_{L} \cdot \bar{V} \\
& D_{T}=\frac{D_{T}}{D_{L}} \cdot \alpha_{T} \cdot \overline{\bar{D}} .
\end{aligned}
$$

$D_{T} / D_{L}$ est appelé le coefficient de dispersion intrinsèque ou dispersivité. Il est homogène à une longueur, et les coefficients de dispersion transversale et longitudinale sont homogènes à une transmissivité. Le contraste qui a été mesuré sur le terrain est de 0,3 à 0,2 .

\subsubsection{Les paramètres}

Il s'ajoute un second phénomène d'échelle. Si l'on mesure les coefficients de dispersion régissant les mouvements d'une dispersion dans une carotte de terrain de $50 \mathrm{~cm}$ de long, ou entre un forage et un piézomètre situé à $5 \mathrm{~m}$, ou encore la pollution dans le champ captant d'Aubergenville, on s'aperçoit que le coefficient de dispersion intrinsèque augmente avec la dimension du problème. De l'ordre de quelques centimètres pour la carotte, il passe aux décimètres pour le forage, et aux mètres pour le problème des captages.

$\mathrm{La}$ raison de ce phénomène est la suivante. La dispersion est créée par les hétérogénéités du terrain, qui font que deux filets de courant très voisins ne peuvent avancer à la même allure, car l'un rencontre des obstacles auquel l'autre échappe. Chaque fois que l'on change d'échelle, les hétérogénéités de l'échelle supérieure surimposent leur effet à celui des hétérogénéités de l'échelle plus fine.

D'autre part, le coefficient de dispersion longitudinale moyen d'une tranche de terrain hétérogène est le résultat d'une intégration qui comporte, en dénominateur, le contraste entre $D_{T}$ et $D_{L}$, ce qui a pour effet d'amplifier le coefficient de dispersion [MARLE, 1967].

Dans le cas présent, comme nous examinons le phénomène à une échelle définie par la maille de $250 \mathrm{~m}$, nous avons pris le coefficient de dispersion dans la gamme correspondante, c'est-à-dire de l'ordre de $10 \mathrm{~m}$. Nous avons observé autour des forages des vitesses de 1 à $10 \mathrm{~m}$ par heure, ce qui a fixé notre gamme de variation des paramètres [DASSONVILle G., et al., 1978].

\subsubsection{Simulation de quelques cas de pollution type}

La simulation de quelques cas de pollution type à l'aide du modèle hydrodispersif, couplé avec le modèle hydrodynamique en régime transitoire, a permis d'observer en premier lieu qu'un arrêt d'urgence des pompages ne présente pas d'intérêt par le fait que le creux piézométrique de la nappe ne se comble pas en quelques heures.

\section{Exemple 1: Simulation d'une pollution par la Seine}

Un accident en Seine revêt un caractère spectaculaire par la rapidité avec lequelle la pollution envahit le champ. Mais en même temps, compte tenu du fort débit du fleuve, il faut des masses très importantes de produit pour atteindre le seuil de $1 \mathrm{ppm}$. En effet, pour une heure de pollution à $1 / 1000$ dans la Seine, il faut, compte tenu d'un débit du fleuve d'environ $300 \mathrm{~m}^{3} \cdot \mathrm{s}^{-1}$ : $300 \times 3600 \times 1 / 1000=1000$ tonnes de produit.

La simulation d'un accident bref d'une heure dans la Seine a permis d'observer l'évolution de la pollution dans la nappe avec un seuil de perception de $1 \mathrm{ppm}$, étant par ailleurs entendu que les pompages ne sont pas arrêtés mais que l'eau est évacuée.

On observe que les forages situés en bord de Seine sont atteints rapidement et que le creux piézométrique est envahi en une semaine. Ultérieurement, l'action des pompages qui évacuent les eaux polluées, font que la pollution est pratiquement résorbée en trois mois (fig. 4), page suivante. 
Scenario : accident bref en Seine en période de hautes eaux, 10 tonnes de polluant déversées en $1 \mathrm{~h}(\mathrm{C}=1 / 1000)$
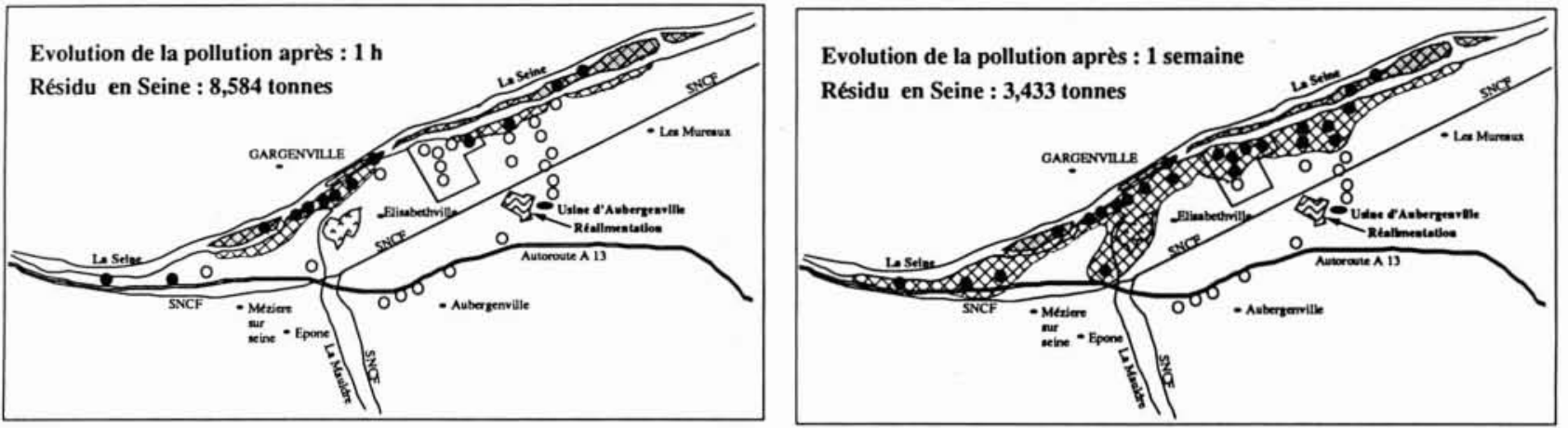

4.

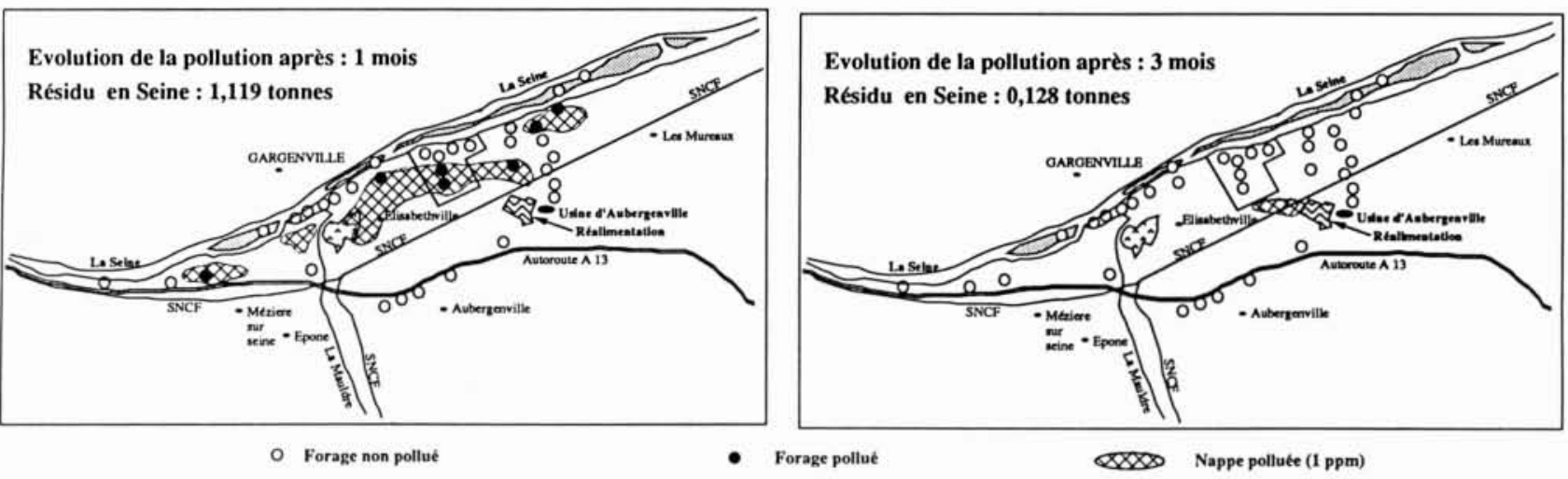

Scenario : accident au lieu dit le Profil (20 tonnes de polluant)
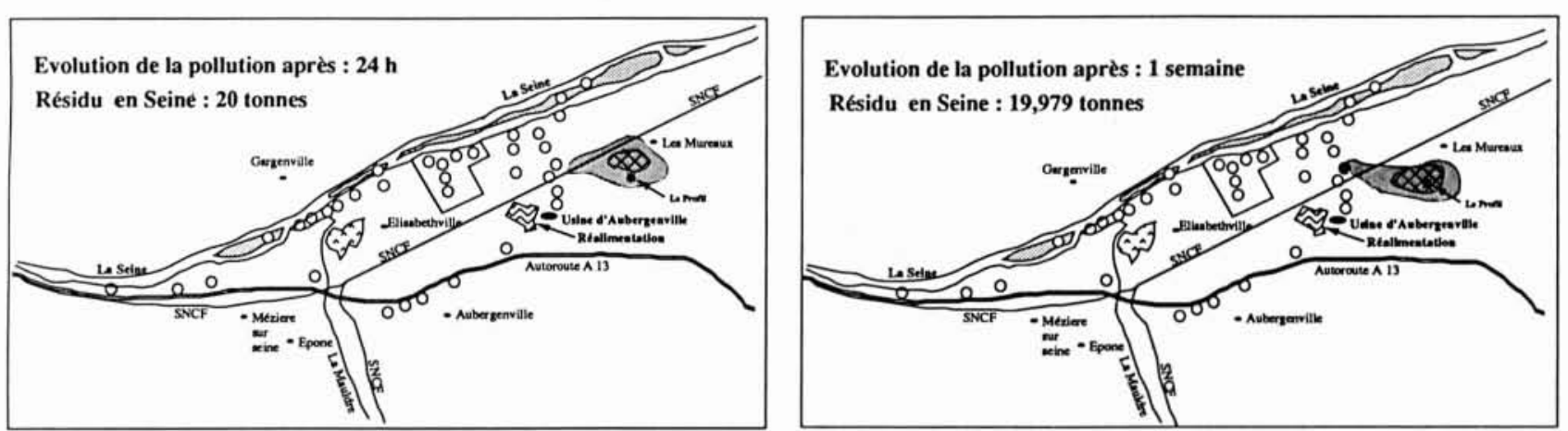

5.
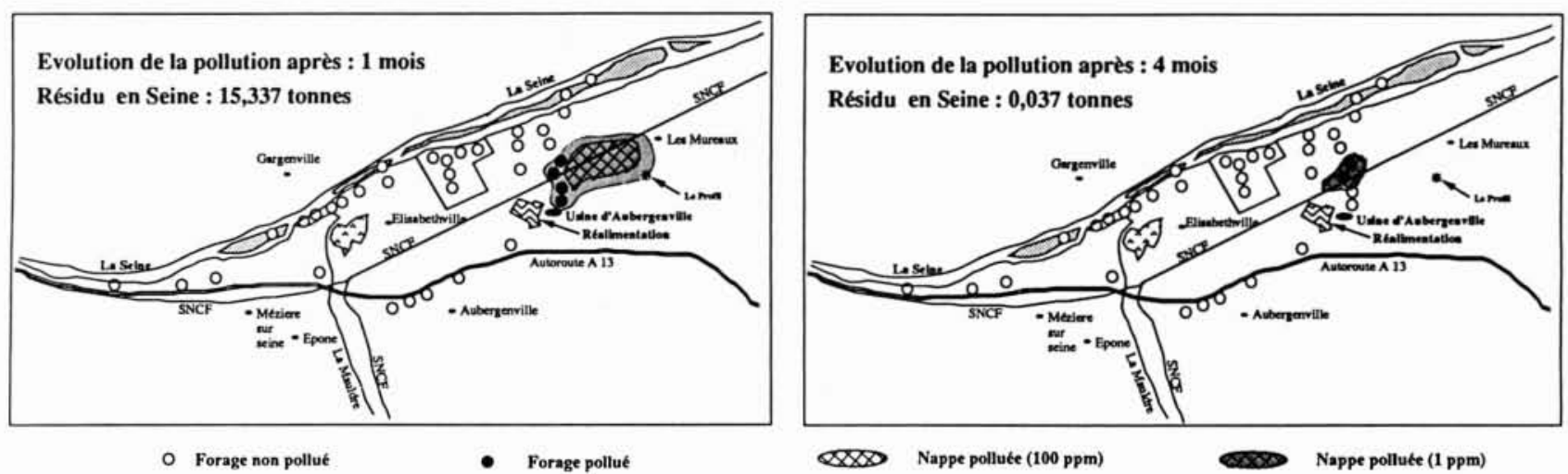

4 et 5. Etude des risques de pollution du champ captant d'Aubergenville. 
Exemple 2 : Simulation d'un accident ponctuel à l'Est du champ captant

On a simulé le déversement de 20 tonnes de polluant au niveau du lieu-dit «Le Profil». Toujours avec un seuil de perception de $1 \mathrm{ppm}$, l'évolution de la pollution dans le temps est représentée dans la figure 5 .

On observe que la pollution ne dépasse pas la première ligne de forages, qu'il ne faut bien sûr pas arrêter mais équiper pour être en mesure d'évacuer l'eau polluée.

La durée d'élimination de la pollution est de l'ordre de cinq mois.

\subsection{Résultats acquis}

L'aquifère de la craie n'est ni homogène ni isotrope [BRGM, 1978]. Les études réalisées montrent que les écoulements se font suivant des axes préférentiels. Les vitesses mesurées mettent en évidence des variations pouvant aller de 20 à $70 \mathrm{~m} \cdot \mathrm{h}^{-1}$ [MEGNIEN C., et al., 1978] dans les axes d'écoulement alors qu'elles ne sont pas mesurables dans d'autres directions. C'est la raison pour laquelle on note des variations importantes des débits d'exploitation pour des ouvrages situés à quelques centaines de mètres les uns des autres (50 à $\left.800 \mathrm{~m}^{3} \cdot \mathrm{h}^{-1}\right)$.

Ainsi il convient de connaître parfaitement la structure de l'aquifère pour implanter les ouvrages de captage et de réalimentation dans les zones présentant les meilleures conditions hydrodynamiques. Par ailleurs il faut suivre en continu les caractéristiques des forages et les volumes infiltrés dans les bassins de réalimentation de manière à pouvoir établir des politiques d'exploitation optimale, de maintenance, et gérer la ressource.

Enfin, l'application de techniques sophistiquées de simulation des écoulements souterrains par modèles mathématiques hydrodynamique et hydrodispersif, permet de cerner macroscopiquement avec une précision suffisante le problème posé par les dangers de pollution.

Les exemples présentés dans le cas d'Aubergenville montrent clairement que si des risques potentiels existent, des solutions permettent de remédier à la situation. L'anticipation de certaines situations critiques permet à l'exploitation d'acquérir des réflexes pour parer à la situation de crise.

\section{Le site du Pecq-Croissy}

Le champ captant du Pecq-Croissy est localisé dans la vallée de la Seine en rive droite, dans un secteur s'étendant de Chatou au Pecq. La nappe est exploitée à raison de $48 \mathrm{Mm}^{3}$ par an au moyen d'une quarantaine de forages à grand débit, gérés par la LED et la Société des Eaux de Versailles et de Saint-Cloud (SEVESC).

Afin de s'assurer du maintien intersaisonnier de la ressource, tant sur les plans qualitatif que quantitatif, la LED procède à une réalimentation artificielle de la nappe. Celle-ci est effectuée grâce à neufs bassins aménagés, à un rythme moyen mensuel de $2 \mathrm{Mm}^{3}$.

\subsection{Cadre hydrogéologique et mécanismes mis en jeu}

L'aquifère est constitué de craie sénonienne surmontée d'une série de couches alluvionnaires récentes et anciennes, dans l'ensemble très perméable, connecté hydrauliquement et pouvant atteindre 10 à $20 \mathrm{~m}$ de puissance. La craie présente généralement un faciès très compact, sauf dans sa partie supérieure dans les secteurs non recouverts par les formations tertiaires, où elle devient fissurée et très perméable. Tel est le cas dans la vallée de la Seine, où elle est également en contact hydraulique direct avec les alluvions sus-jacentes. Le lit de la Seine ne constitue pas une limite d'extension de la nappe puisqu'il ne recoupe que partiellement les couches perméables.

Ces quelques remarques permettent de fixer les limites latérales du modèle aux limites d'extension du recouvrement tertiaire. Les limites amont et aval, en revanche, ne correspondant à aucun argument structural, géologique ou hydrogéologique, ont dû être repoussées suffisamment loin des zones d'intérêt pour éviter les effets de bord.

Les principaux mécanismes hydrodynamiques mis en jeu dans cette zone sont, pour l'exhaure, les prélèvements, et pour les apports, les échanges Seine-nappe, la réalimentation artificielle, et dans une mesure nettement moindre, les infiltrations pluviométriques et les transferts latéraux par les limites.

Les échanges Seine-nappe dépendent d'une manière générale de la largeur de sections mouillées des bras, et de l'épaisseur et nature des sédiments fins colmatant son lit. Dans le cas de Croissy, ces deux facteurs sont fonction d'une série de phénomènes ou événements parfois non linéaires, évolutifs dans le temps et dans l'espace comme, par exemple, la distribution géographique des zones inondées et des précipitations, la nature et l'intensité des précipitations, l'état initial du système, l'effet d'écluse, ou encore la gestion des barrages sur le réseau hydrographique amont.

Enfin, la nappe de Croissy peut être dans l'ensemble considérée comme ayant une surface libre.

\subsection{Les modèles hydrodynamiques}

En 1979 et 1980 ARLAB a procédé à l'élaboration d'un premier modèle mathématique de la nappe à grande échelle. Cette première étude a permis :

- de préciser l'importance relative des différents flux d'eau ;

- de cerner avec précision le rôle joué par la réalimentation artificielle sur le comportement de l'aquifère ;

— de tester différentes solutions envisageables pour augmenter la capacité de la réalimentation ;

- de calculer la vitesse de remontée de la nappe après l'arrêt des pompages en cas de pollution grave de la Seine.

En 1988, compte tenu des résultats obtenus par le premier modèle et de l'importance économique de l'aquifère, la LED a confié à SAFEGE l'élaboration d'un outil permettant de gérer au mieux la ressource en eau en fonction des objectifs de prélèvement et des contraintes et impératifs liés à l'exploitation. Ceci concernant notamment la réalimentation dont le coût reste 
onéreux et l'exploitation "empirique ». L'outil de gestion confié à SAFEGE consiste en l'élaboration de trois modèles distincts :

— un modèle numérique d'écoulement souterrain,

- un modèle prévisionnel de la capacité de transfert Seine-nappe,

— un modèle d'optimisation.

Ces modèles sont ensuite intégrés en un seul modèle de gestion doté d'une interface conviviale.

\subsubsection{Le modele numérique}

Les écoulements de la nappe du Pecq-Croissy sont représentés par l'équation suivante :

$$
\begin{aligned}
\frac{\delta}{\delta x}\left(T_{x} \frac{\delta h}{\delta x}\right)+\frac{\delta}{\delta y}\left(T_{y} \frac{\delta h}{\delta y}\right)+ & Q+Q_{s}+ \\
& +Q_{l}+Q_{m}=S \frac{\delta h}{\delta t}
\end{aligned}
$$

avec :

$h \quad$ potentiel hydraulique

$S \quad$ coefficient d'emmagasinement

$Q \quad$ somme algébrique des débits prélevés ou injectés par unité de surface

$Q_{s} \quad$ débit par unité de surface échangé avec la Seine avec $Q_{s}=c\left(h_{s}-h\right)$

$Q_{1} \quad$ apport par unité de surface aux limites du domaine

$Q_{m} \quad$ échange avec l'atmosphère par unité de surface c coefficient de diffusibilité du lit de la Seine

$h \quad$ potentiel de l'aquifère

$h_{s} \quad$ potentiel de la Seine

$T_{x}$ et $T_{y}$ transmissivité selon $x$ et $y$, avec $T=\int_{q}^{e} K \mathrm{~d} x$ et $e=$ épaisseur et $k=$ perméabilité

L'équation (2) traduit la conservation du stock d'eau dans une unité de volume de l'aquifère. Le membre $S \delta h / \delta t$ représente la variation de stock d'eau dans cette unité de volume ; cette dernière est liée aux fluctuations piézométriques dans le temps.

Ces équations sont résolues, sur calculateur, dans un espace discret, en différences finies. Le modèle a pour principal objectif de simuler les écoulements souterrains dans les différentes couches aquifères, en intégrant les échanges avec l'extérieur (nappe-Seine, pluviométrie, conditions aux limites, etc.). Il sert également de base pour le calcul de la répartition optimum des prélèvements et de la réalimentation artificielle. Il comporte 1679 mailles avec de grandes mailles de $200 \mathrm{~m}$ de côté, des mailles moyennes de $100 \mathrm{~m}$ de côté, des petites mailles de $50 \mathrm{~m}$ de côté et des micro-mailles de $25 \mathrm{~m}$ de côté. Les deux dernières servant à étudier les zones sensibles (champ captant, réalimentation).

$\mathrm{Au}$ niveau du bilan des flux on remarque qu'en moyenne, pendant les onze dernières années :

- l'ensemble des réalimentations et prélèvements s'équilibrent autour de $14451 . \mathrm{s}^{-1}$,

- la réalimentation de l'aquifère est assurée par: la Seine pour $50 \%$, les sablières de réinjection LED pour $37 \%$, les limites latérales pour $9 \%$, les apports météoriques pour $4 \%$.

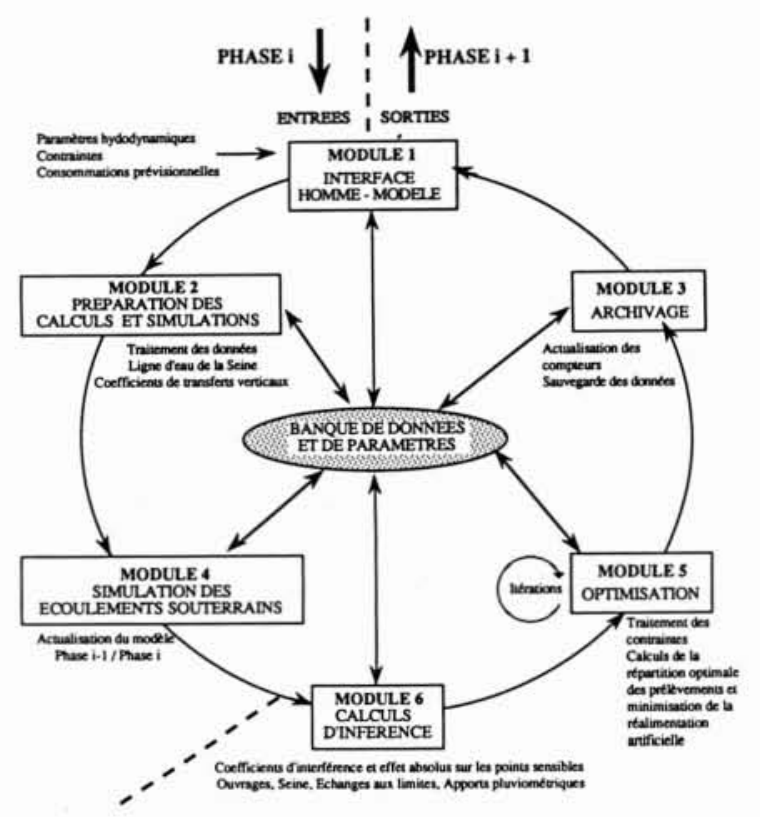

6. Synoptique du fonctionnement de SOPHOS (modules de base).

L'analyse de détail du modèle met en évidence que la majorité des excédants de recharge vers la nappe prend place pendant les mois froids alors que les déficits interviennent pendant les mois chauds. Ce dernier résultat nous a notamment conduits à développer le modèle de gestion optimale.

\subsubsection{Le modèle prévisionnel de transfert Seine-nappe}

L'objet du modèle prévisionnel est de pouvoir calculer les coefficients de transfert verticaux en fonction d'un paramètre facilement mesurable : le niveau de la Seine. Le problème revient à identifier un opérateur qui, appliqué sur l'historique des niveaux de la Seine, permet de calculer l'évolution des coefficients verticaux dans les trois secteurs utilisés pour représenter son lit.

Il est apparu que le modèle de transfert Seine-nappe est le point le plus fragile du modèle global [SAFEGE, 1988]. En effet, l'étude des crues de 1987 et surtout 1982 montre que le modèle est subordonné aux crues. Les crues doivent entraîner une partie du matériau de colmatage, ce qui facilite alors le transfert de la Seine vers la nappe. Ce nouvel état aux limites nécessite alors un rétalonnage du modèle de transfert pour que le modèle global puisse coller à la réalité.

\subsubsection{Le modele d'optimisation}

L'objectif du modèle d'optimisation consiste à intégrer sur le modèle hydrodynamique un modèle de gestion, de manière à minimiser le coût de production de l'eau potable.

La problématique revient à appréhender en continu la piézométrie de la nappe, en fonction de l'évolution dans le temps des conditions aux limites (précipitations, niveau de la Seine, évapotranspiration, réalimentation, prélèvements, etc.) et de dégager des consignes d'exploitation minimisant les coûts (notamment les consommations d'énergie et la réalimentation dont le coût intervient pour une partie significative dans le prix de revient du mètre cube d'eau distribué). 


\subsection{Le modèle de gestion : SOPHOS}

Le modèle global réunissant les trois sous-modèles précédents répond au nom de SOPHOS. Il comprend également une banque de paramètres et de données et une série d'interfaces et de logiciels de calculs intermédiaires [PENNEQUIN D., et al., 1991] (fig. 6).

Ces modèles, couplés les uns aux autres, permettent d'étudier des phénomènes complexes. Cette approche a eu pour conséquence de faire naître une nouvelle conception du modèle qui n'apparaît plus comme le support d'une étude mais devient la partie intelligente du tableau de contrôle de la nappe.

Le modèle et les interfaces ont été écrits en Fortran 77 ou sous forme de procédure de commande DCL (Digital Command Langage). Il est installé sur un Micro Vax 2 et fonctionne dans un environnement VAX sous VMS (marques déposées de Digital Equipment Coorporation).

\subsection{Résultats acquis}

Le modèle intégré de gestion optimale de la nappe de Croissy fonctionne depuis mai 1988 à la LED. La première année a servi de test, ce qui a permis de vérifier son fonctionnement par rapport au système réel.

Depuis sa mise en service, SOPHOS a conduit à diminuer significativement les coûts mensuels d'exploitation et a permis une prise de conscience de la gestion de la ressource.

La principale retombée économique se situe au niveau de la gestion de la réalimentation en permettant une optimisation de l'utilisation du système complexe « réalimentation-exploitation ». Par ailleurs, en proposant des solutions optimales chaque mois, SOPHOS a fait naître, chez l'exploitant, à tous les niveaux de la hiérarchie, une prise de conscience du fonctionnement du système hydrologique. Ceci a notamment permis de remettre en question certaines pratiques « antiéconomiques » utilisées de longue date par habitude. Enfin il a permis d'obtenir une vision du patrimoine hydrogéologique, par le biais de cartes isopièzes, d'exploitation, de réalimentation, etc., véritables éléments d'appréciation concrets de l'état de santé de la nappe.

Ces éléments donnent à l'exploitant le moyen de maîtriser l'évolution de sa ressource en permettant la définition du cadre d'exploitation plusieurs semaines à l'avance, et ainsi de devancer les événements. Le modèle permet une sécurité accrue vis-à-vis des consommateurs en maintenant une ressource capable de satisfaire les demandes. Cet aspect a été particulièrement apprécié en 1989 et 1990, années relativement sèches pendant lesquelles une bonne anticipation des phénomènes a permis d'assurer sans incidents l'approvisionnement en eau du secteur.

Enfin, il est prévu d'étendre le modèle à la prise en compte de l'aspect qualitatif. En effet, la qualité de la ressource au niveau des champs captants peut être soumise à des variations spatiales et temporelles, ce qui conduit à faire fluctuer le prix du traitement. C'est un facteur qui peut être significatif dans certains cas, et particulièrement lors du passage de pollutions accidentelles dans la Seine.

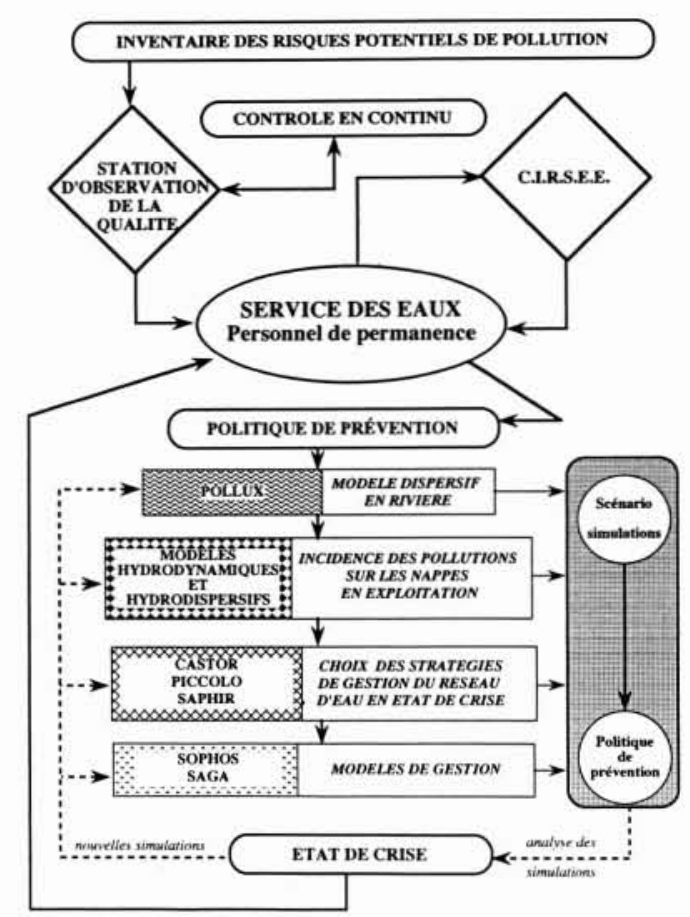

7. Gestion du risque de pollution.

\section{Les outils de la sécurité}

Jusqu'à la dernière décennie, l'accroissement considérable des besoins fut un facteur primordial qui orienta l'essentiel des investissements. Plus récemment, on a pu mettre à profit une détente dans cette course à la production pour s'attacher à améliorer la sécurité de l'approvisionnement et à réduire la vulnérabilité du système d'alimentation.

Les risques de défaillance de l'approvisionnement en eau de l'agglomération parisienne sont de trois ordres :

- quantitatif: révélé lors de la sécheresse de 1976;

- qualitatif: il s'agit de pouvoir respecter des normes sévères de qualité des eaux destinées à la consommation humaine malgré des concentrations parfois croissantes en produits polluants dans les eaux superficielles. Les nombreuses modifications apportées ces dernières années aux filières de traitement réduisent déjà considérablement ce risque ;

- occasionnel : dû à des événements ponctuels, ou sur l'une des trois rivières nourricières de la région parisienne (Seine, Marne, Oise). Les études entreprises dans ce sens ont permis de définir des solutions techniques: intercommunications de réseaux, bassins de stockage, réserves d'eau traitée, etc., en fonction des risques potentiels de pollution.

Depuis peu, le milieu des distributeurs d'eau est sensibilisé au risque de pollutions accidentelles des eaux brutes. Quelques exemples en France ont montré la réalité de ce risque et mis l'accent sur l'ampleur des conséquences possibles sur la sécurité civile [D'ARRAS et al., 1990].

Il n'y a pas d'outil miracle mais une panoplie d'instruments. La politique de gestion mise au point comporte quatre volets complémentaires, de la prévention à la résolution du problème (fig. 7). 


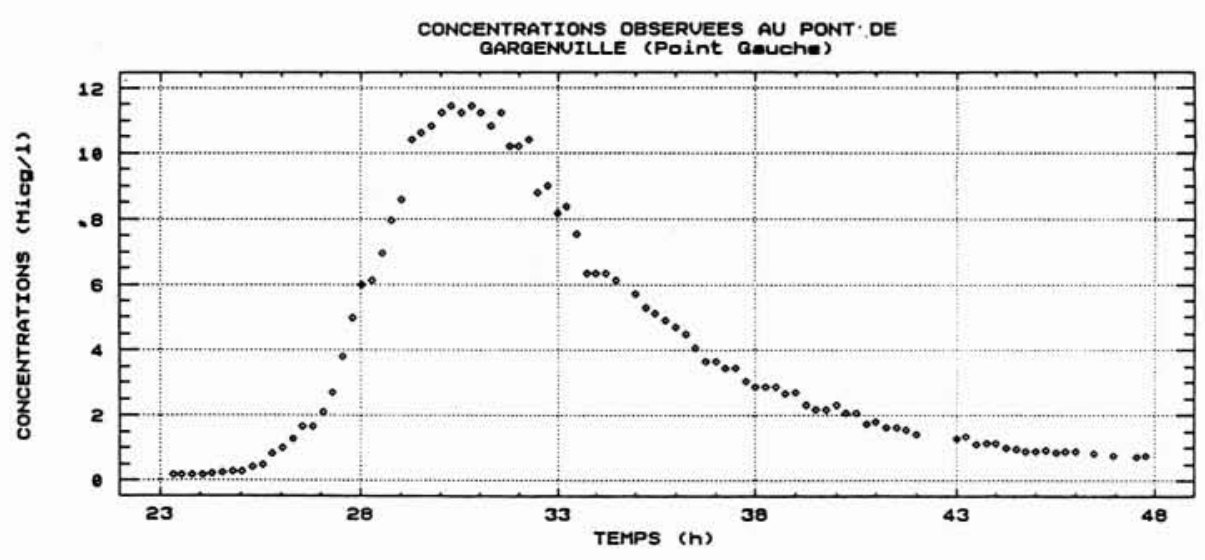

8. Pollutogramme obtenu par traçage en Seine (Rhodamine B).

\subsection{Politique préventive : inventaire des risques potentiels de pollution}

L'étude de sensibilité d'un site à la pollution, avec un recensement systématique des pollueurs et polluants potentiels, s'avère indispensable en milieu urbain ou industriel.

Ces études doivent être menées en collaboration étroite avec le Service des Mines dans la mesure où les industriels se retranchent souvent derrière le secret professionnel. La carte géographique des implantations industrielles, des réseaux d'assainissement de routes et voies ferrées met en valeur la nature et l'ampleur du risque. La prise de conscience induite au niveau des différents partenaires (Industriels, Service des Mines, Service de la Navigation, Distributeurs) et les contacts que l'on peut nouer à l'occasion de la réalisation de ce recensement rendront plus facile en cas d'incident la communication des informations importantes (nature des polluants, quantité) éléments primordiaux pour évaluer rapidement l'impact d'une pollution.

Enfin, le recensement permet d'orienter le choix des capteurs à installer dans les stations d'observation de la qualité des eaux brutes. Le recours de plus en plus fréquent aux stations d'alerte sur les eaux de rivière, et même sur les eaux de nappe, se justifie amplement pour des raisons de sécurité. Tout poisson mort sur la rivière ne signifie pas forcément risque pour la distribution de l'eau ; il est important de pouvoir faire la part des choses rapidement, et de pouvoir déclencher des analyses complémentaires le plus vite possible.

\subsection{Contrôle en continu : les stations d'observation de la qualité de l'eau}

Ces stations, véritables vigies de la qualité de l'eau, ont plusieurs rôles :

- surveiller et donner l'alerte en cas de dégradation subite de cette qualité ;

- acquérir en permanence une connaissance complète et actualisée de l'état des rivières et des nappes souterraines ;

— éventuellement évaluer l'impact d'une politique d'assainissement.
Les paramètres de la qualité des eaux sont choisis après identification des risques potentiels de pollution dans le secteur d'implantation de chaque station.

En Région Parisienne, la LED assure ainsi un contrôle permanent de la qualité des eaux de la Seine au moyen de deux stations (stations de Nandy et du Mont Valérien), mais aussi de la qualité des eaux souterraines à l'aide d'une station installée sur le champ captant d'Aubergenville.

D'une façon générale les principaux paramètres, analysés simultanément, sont : $\mathrm{pH}$, température, oxygène dissous, conductivité, turbidité, hydrocarbures, ammonium, matières organiques, métaux lourds, phénols. Ces stations comportent en outre la détection de toxiques par activité piscicole. A l'amont de Paris, à Nandy, une mesure permanente de la radioactivité $\alpha$ et $\gamma$ a été rajoutée à cette chaîne, en raison de la présence de l'usine nucléaire de Nogent-sur-Seine. A Aubergenville, les paramètres retenus et analysés par la station ont été déterminés compte tenu de l'environnement du champ captant : hydrocarbures, ammonium, métaux lourds, matières organiques, couleur, solvants organiques...

Les stations d'observation de la qualité des eaux de la LED sont coordonnées, en Région Parisienne, par l'AFBSN, et s'intègrent dans un réseau qui regroupe l'ensemble des distributeurs (CGE et SEDIF, SAGEP, CEB, LED).

Dans le cadre d'une stratégie de gestion du risque de pollution, l'utilisation des stations d'observation de la qualité de l'eau donne un moyen supplémentaire pour anticiper l'arrivée d'une pollution et permet de s'organiser pour y faire face. La détection déclenche toute une série de vérifications qui sont effectuées très rapidement par le CIRSEE : prélèvements et analyses confirmeront ou infirmeront la réalité du danger.

\subsection{L'évaluation de l'impact de l'incident : l'utilisation des modèles}

Il est fondamental d'avoir en permanence à sa disposition des outils permettant de mesurer l'intensité et la vitesse d'arrivée d'une pollution sur les ressources en eau brute. En fait, il est important de s'être préparé à une pollution, d'avoir évalué son impact, de s'être forgé des réflexes à froid pour que le jour $J$ les mécanismes d'intervention soient rodés. 
Pour ce faire, la LED a développé non seulement des modèles de nappe mais aussi un modèle hydrodynamique et hydrodispersif de pollution en rivière. En effet, sauf si la pollution a lieu au droit de la prise d'eau, il est indispensable de pouvoir évaluer le temps de transfert d'un polluant entre l'amont et l'aval et l'effet de dispersion qui agira sur la concertation et la durée de l'incident.

C'est ce à quoi répond le modèle «POLLUX " développé par la SAFEGE modélisant la Seine sur $120 \mathrm{~km}$ de Morsang à Aubergenville. Ce modèle a été calé à partir de campagnes de traçage en Seine (fig. 8).

\subsection{L'aide à la résolution de l'incident : utilisation de I'informatique avancée}

Heureusement les opérateurs habituels des dispatchings de grands réseaux d'eau ont peu d'expérience d'incidents majeurs. Leur mode de raisonnement s'applique alors mal aux situations extrêmes. C'est la raison pour laquelle trois outils informatiques ont été développés et font maintenant partie intégrante de la panoplie des outils dont dispose la LED en Région Parisienne Ouest, pour une gestion optimale de la ressource en eau en cas de pollution accidentelle.

\subsubsection{Le système expert "CASTOR "}

Développé par le Laboratoire d'Informatique Avancée de Compiègne (LIAC), «CASTOR » a été réalisé pour gérer les échanges d'eau. Ce programme permet de simuler des arrêts majeurs d'installations de production d'eau (arrêt total ou partiel d'une ou de plusieurs unités de distribution), et de proposer des solutions alternatives pour trouver des volumes d'échanges nécessaires aux interconnexions de sécurité et pallier aux incidents. Le système est basé sur une modélisation simplifiée du réseau établi par les ingénieurs chargés de la production de chaque unité en vue de prendre en compte les caractéristiques de distribution, de consommation et de réserve utiles. Il intègre les éléments, les relations et les contraintes qui caractérisent les unités de production (capacité de production et de stockage, caractéristiques structurelles du réseau, contraintes de sécurité, échelles de priorité de distribution, etc.). Le système expert utilise alors l'ensemble des éléments décrits pour :

- reconfigurer le réseau en fonction des éléments perturbateurs engendrés par la crise,

- générer dans le temps toutes les solutions possibles d'échanges et de gestion des ressources disponibles, - estimer et quantifier les échanges entre les blocs du modèle, de manière à " passer " au mieux l'événement.

La mise en œuvre très rapide de CASTOR en fait un excellent outil d'aide à la décision en périodes de crise. Ce type d'outil s'est avéré très pédagogique pour le personnel d'astreinte qui en simulant quelques cas d'école a pu découvrir les larges possibilités de dépannage qu'offrent les réseaux interconnectés.

\subsection{2. "PICCOLO Qualité "}

Un nouvel outil, mis au point par la SAFEGE, surimpose au modèle hydraulique du réseau de distribution «PICCOLO » un module qualitatif. Sur la partie classi- que du réseau maillé, a été ajouté un module de qualité dynamique qui permet de suivre le chemin de l'eau. On sait ainsi simuler l'expansion éventuelle d'un produit dans le réseau. L'utilisation fréquente de ce modèle concerne, à la base, la cinétique d'évolution du chlore résiduel, mais il est possible de simuler tout autre molécule chimique. Là encore, l'usage fréquent du modèle permet de réagir à bon escient en cas de nécessité.

\subsection{3. "SAGA "}

Le système expert « SAGA » est un système d'aide à la gestion des alarmes. Il fournit aux responsables d'un contrôle centralisé les éléments d'information et de décision concernant une alarme quelle que soit l'origine et l'importance de celles-ci. En assurant ainsi la diminution, voire la disparition des mauvaises interprétations d'alarme, SAGA accroît la sécurité des installations et spécialement les jours de forte intensité émotionnelle que sont les jours de crise.

\subsection{4. " SAPHIR "}

SAPHIR est un outil d'aide à l'exploitation d'un réseau de distribution d'eau potable. Il a été conçu pour apporter une aide à la décision aux dispatcheurs dans leur travail quotidien. Il permet de rechercher un profil optimal de gestion et de l'adapter aux évolutions réelles de la situation dans la journée.

SAPHIR fournit, par tranches horaires, les volumes à produire et les débits à transférer (consignes) et détermine les niveaux de bâches et des réservoirs (état du réseau). Les échanges sont décrits à partir de débits et les pressions sont prises en compte à travers la définition des contraintes. Les calculs intègrent les coûts d'exploitation, les objectifs stratégiques et les heuristiques (savoir-faire) ainsi que les critères de sécurité de fonctionnement.

Une première application de SAPHIR a été réalisée pour le réseau de RPO. L'application a été implantée sur une Vax Station 3100 et a fourni des résultats de calcul comparables à ceux des dispatcheurs (temps de réponse d'environ $2 \mathrm{mn}$ ). Au vu de la qualité des résultats obtenus, la LED envisage d'étendre le système à d'autres sites.

L'ensemble des modules de calcul a été développé en $C$, tandis que les interfaces ont été écrites en LELISP utilisant les logiciels AIDA et MASAI. L'architecture générale du système est représentée en figure 9.

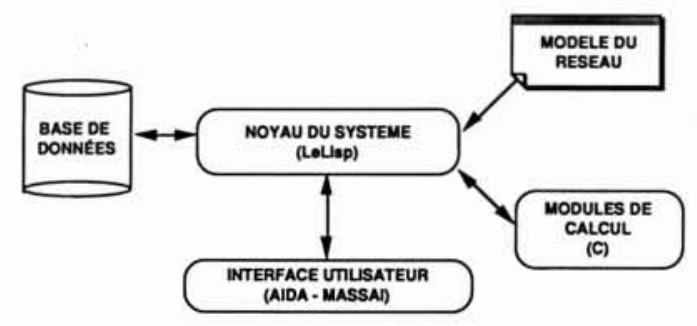

9. Architecture générale du système SAPHIR. 

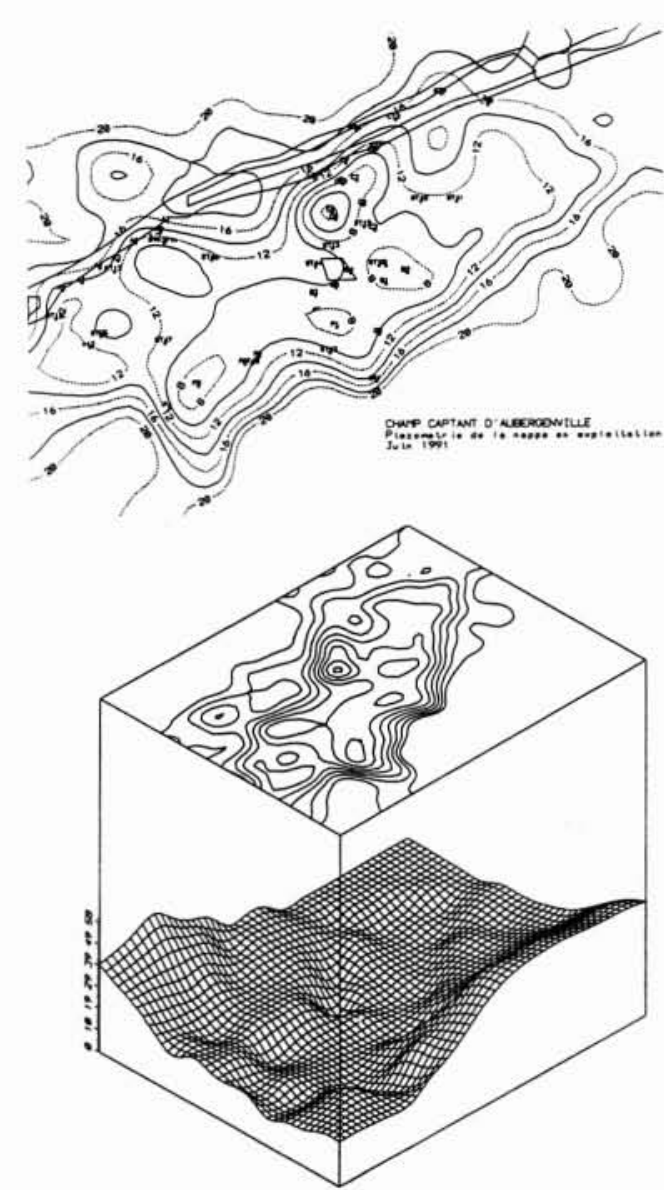

10. Champ captant d'Aubergenville en exploitation: carte isopièze en plan et représentation $3 D$ à $225^{\circ}$ avec une incidence de $35^{\circ}$.

\subsection{Résultats acquis}

Dans le domaine des outils de la sécurité, on ne saurait trop insister sur l'utilisation de l'image (image « $3 D$ », visualisation des niveaux piézométriques, des actions de réalimentation, des panaches de pollution, etc.) pour favoriser une meilleure prise de décision plutôt qu'une sortie informatique abstraite [DETAY M., 1991]. Il nous semble important de développer l'utilisation de la troisième dimension car la notion de profondeur est mal perçue par ceux qui n'ont pas l'habitude d'interpréter l'espace. Nous sommes à l'heure de l'audiovisuel et nous nous devons de nous orienter vers l'image de synthèse. Dans cet esprit la représentation en plan et en $3 D$ (fig. 10) de la piézométrie d'un champ captant en exploitation, permet une appréciation instantanée de phénomènes souvent complexes. Elle doit être axée sur les zones sensibles étudiées et favoriser la prise de décision. Ces outils donnent une vision de l'aménagement de l'espace. Ils permettent aux décideurs de se rendre compte des circulations de l'eau souterraine et des biens qui la surmontent. Enfin l'imagerie doit être couplée à des systèmes d'alerte intégrant les aspects techniques mais aussi géographiques (SIG) et les dispositions légales (DUP, POS, schéma d'aménagement, etc.).
Il devient alors possible d'appréhender et d'agir sur ce qu'il convient désormais d'appeler le "paysage hydrogéologique " dans le cadre d'une politique d'aménagement du territoire.

Enfin, il est nécessaire de se préparer à la gestion de crise de pollution et de réaliser des exercices préparatoires de manière à être prêts et à réagir à bon escient.

\section{Des actions de recherche appliquée}

Les enseignements tirés montrent que la méconnaissance de l'hydrogéologie ainsi que du rôle d'avant-garde des moyens modernes d'action sur les eaux souterraines entraînerait vite un état de sous-développement ou de sous-exploitation des ressources en eau.

Différents volets restent encore méconnus et la LED participe à des travaux de recherche appliquée, aspect fondamental indispensable à la compréhension des phénomènes, et vise, à terme, à la mise en place d'une politique de maintenance active pour ses champs d'exploitation. Cette dernière implique une meilleure connaissance de l'hydrogéochimie et des phénomènes connexes; le développement et la coordination de compétences locales, nationales et internationales dans plusieurs disciplines de la géologie, de l'hydrogéologie, de la géochimie, de la géophysique ; appelle, enfin, à se placer résolument dans la réalité de l'espace scientifique européen, pour l'industrie et pour la recherche.

Nous résumons, dans ce paragraphe, les principaux axes de recherche ayant trait à l'hydrogéologie quantitative.

\subsection{Les échanges rivière-nappe}

Un travail de recherche, subventionné par l'Agence de l'Eau Seine-Normandie, est actuellement en cours, il a pour objectif d'étudier dans le détail le transfert de polluants entre une rivière et la nappe alluviale connectée. En effet, une grande partie de l'eau produite en France provient des nappes alluviales réalimentées par des cours d'eau. Cette dépendance de la nappe vis-à-vis du cours d'eau amène plusieurs questions :

- Quelles sont les répercussions sur la qualité de la nappe en cas de pollution chronique ou accidentelle de la rivière?

- Quels sont les mécanismes gouvernant le transfert de ces polluants à l'interface rivière-nappe ?

Après avoir réalisé une revue bibliographique des phénomènes de transfert rivière-nappe [DousSAN C., 1989], il a été possible de dégager quelques points clefs sur le comportement du système :

- Au cours du trajet des polluants, les principaux mécanismes mis en jeu sont : la dispersion, l'adsorption, la décroissance radioactive, les réactions en solution, et la dégradation bactérienne.

- L'élimination des polluants semble se dérouler sur les premiers mètres de berges. Parmi les composés bien éliminés, on dénote: la matière organique dissoute, certains métaux : $\mathrm{Ag}, \mathrm{Hg}, \mathrm{Cr}, \mathrm{Cd}, \mathrm{l}$ 'ammonium et une 
partie des nitrates, les hydrocarbures... D'autres composés sont peu éliminés et peuvent se retrouver dans les captages : halométhanes, composés organiques chlorés, pesticides (atrazine).

- Les caractéristiques chimiques générales sont réglées par l'oxydation de la matière organique par les bactéries ; on assiste à une consommation de l'oxygène dissous, des nitrates, des sulfates et parfois du $\mathrm{CO}_{2}$. Il existe donc une zone réductrice s'étendant sur une certaine longueur de berge, dépendant des possibilités de réaération de la nappe par diffusion de l'oxygène à partir du sol. L'ensemble de ces mécanismes interfère avec l'équilibre calcocarbonique.

Il était cependant nécessaire de vérifier les cinétiques des différents mécanismes, et de mener des études en laboratoire et in situ pour quantifier les différents phénomènes. Trois familles de polluants ont été retenues dans le cadre de cette étude :

- l'azote, du fait de l'augmentation sensible des teneurs en nitrates dans les cours d'eau, en particulier en Seine en aval de la station d'épuration d'Achères ;

- des radioéléments, issus du fonctionnement normal d'une centrale nucléaire ( $\mathrm{Co}, \mathrm{Cs}, \mathrm{Ag}, \mathrm{Sb}$ ) ou résultant d'un accident (I, Sr, Cs, Ru, Te) ;

- des pesticides (Atrazine et Simazine) dont il a été détecté des concentrations importantes au printemps dans les aquifères et les cours d'eau des zones cultivées.

Le programme technique retenu comprend trois parties :

- un suivi analytique sur le terrain grâce à l'implantation de piézomètres sur le trajet de l'écoulement rivièrenappe-forage ;

— l'acquisition de paramètres intrinsèques au système par des expériences en laboratoire (transformations biologiques des polluants, adsorption, ...);

- la synthèse des données recueillies et l'identification des phénomènes importants qui devront permettre d'établir un modèle de transfert des polluants.

Les premiers résultats montrent une activité biochimique importante dans les premiers centimètres de boues de Seine ainsi qu'une épuration notable dans les premiers mètres de berge [Doussan C., et al., 1991]. De plus, l'eau de la nappe aurait des origines différentes selon la profondeur et la distance par rapport à la Seine (différences dans les piézomètres en doublets et anomalie systématique du piézomètre à $20 \mathrm{~m}$ de la berge). D'autre part, il apparaît une «bulle » de concentrations élevées en $\mathrm{P}, \mathrm{NH}_{4}^{+}, \mathrm{Fe}$ au niveau des boues de Seine ; il importe de connaître la façon dont ces concentrations vont évoluer.

\subsection{Optimisation de la réalimentation artificielle}

La réalimentation artificielle vise à un accroissement de la ressource et consiste à recharger la nappe de la craie pour compenser l'effet de la dépression piézométrique liée à l'exploitation intensive de l'aquifere. Ce procédé de « gestion rationnelle d'un réservoir naturel " permet de stocker dynamiquement des ressources et de les réutiliser dans des conditions de régime et de qualité différente.
A Aubergenville l'alimentation artificielle de la nappe de la craie sénonienne et des alluvions est réalisée à partir des eaux de Seine traitées par l'intermédiaire de bassins aménagés dans d'anciennes sablières. Ces bassins situés dans la zone de captage sont au nombre de 6 . Leur superficie totale est de 4 ha. Une "couche filtrante» constituée de sables a été mise en place dans le fond des sablières. La capacité unitaire d'infiltration est de l'ordre de 0,4 à $1 \mathrm{~m} \cdot \mathrm{j}^{-1}$ avec une hauteur d'eau de $2 \mathrm{~m}$.

Une étude d'optimisation de la réalimentation artificielle est actuellement en cours. Elle a pour but d'identifier les causes de colmatage des bassins et de définir une politique de maintenance qualitative et quantitative. En effet, l'adaptation d'un projet d'aménagement aux conditions hydrogéologiques du site n'est qu'un préalable : il reste à étudier le colmatage, les moyens de le limiter, et toutes les conséquences sur l'entretien, dont les incidences financières pèsent sur la rentabilité de l'exploitation.

Le colmatage des bassins résulte principalement de la prolifération des algues, du dépôt de matières en suspension et des actions bactériennes sur les terrains. On note compte que bien que la technique de réalimentation par bassin soit assez répandue, sans doute en raison de l'apparente simplicité du procédé, son emploi, examiné de près, est fort complexe, dès que l'on cherche à se placer dans des conditions d'exploitation optimales.

Tous ces travaux s'inscrivent dans la logique d'une politique de gestion des ressources en eau. Elle a pour but, à travers une meilleure connaissance des aquifères, de définir des actions de maintenance (développement des forages avec des acides passivés, étude de colmatage bactérien, etc.).

\section{Conclusions}

Les auteurs montrent, à travers la description de l'environnement de deux champs captants en Région Parisienne Ouest, l'aspect fondamental de la gestion de la ressource, tant en son gîte souterrain que lors de son trajet vers l'abonné.

Ils dégagent les grandes étapes de la démarche de la LED pour intégrer ses travaux au sein du fonctionnement des écosystèmes aquatiques, en regroupant les aspects eaux souterraines-eaux superficielles-eaux de distribution, dans une action visant à préserver l'environnement.

Grâce à cette approche, de nouveaux axes de recherche appliquée ont émergés : compréhension des transferts rivière-nappe, techniques de réalimentation artificielle des aquifères, optimisation de l'exploitation des champs captants, meilleure connaissance des phénomènes physico-chimiques et biologiques et de leur évolution dans le cycle de l'eau.

On perçoit bien, dans nos sociétés modernes, que le formidable enjeu représenté par la maîtrise de l'eau, exige des réponses de plus en plus fines. L'eau souterraine est un élément essentiel de cet enjeu. Ceci souligne toute l'importance des travaux présentés dans cette rapide synthèse. Ils sont le résultat d'un savoir-faire considérable qu'il faudra encore accroître pour pouvoir demain posséder des outils à la hauteur de cet enjeu. 


\section{DETAY et al.}

\section{Références bibliographiques}

ARLAB, 1979-83. - Etude sur modèle mathématique de la nappe de Croissy. Rapports finaux $\mathrm{n}^{*} 140 / 81,162 / 82$, $182 / 83$.

ARLAB, 1976-77. - Gestion de la nappe d'Aubergenville par modèle mathématique de la nappe d'Aubergenville. Rapports finaux $n^{\circ} 056 / 77$ et $065 / 77$.

AFBSN, Direction de I'Industrie d'Ile-de-France, Lyonnaise des Eaux, 1986. Etude des risques de pollution de la nappe d'Aubergenville.

Berger G., Megnien C., et al., 1975. - Origine et bilan des eaux souterraines prélevées dans la région d' Aubergenville-Porcheville (Yvelines). Rapport BRGM n*75 SGN 275 BDP.

BRGM, 1982. - Etude de l'environnement et du contexte hydrogéologique et technique des champs dits de Croissy. Rapport $n^{\circ} 82$ SGN 877 IDF.

BRGM, 1978. - Hydrogéologie de la craie du bassin de Paris. Actes du colloque régional de Rouen 25-26 mai. Doc. $B R G M, \mathrm{n}^{\circ} 1,627 \mathrm{p}$. et $\mathrm{n}^{\circ} 3,155 \mathrm{p}$.

CPGF, 1985. - Etude de la vulnérabilité du champ captant d'Aubergenville. Evaluation des vitesses d'écoulement et des propriétés dispersives de la nappe. Etudes n`2509 et 2633.

D'Arras D., Demongeot D., Loubeyre R. - Des outils de sécurité en matière de distribution d'eau potable. TSM $L^{\prime} e a u, \mathrm{n}^{\circ} 10$, pp. 489-490.

D'Arras D., Suzanne P., 1991. - Protection des ressources en eau, aspects légaux et opérationnels. IWEN Annual Symposium.

Dassonville G., Suzanne P., 1978. - L'étude par modèle mathématique de la nappe d'Aubergenville. TSM L'eau, pp. 259-267.

Detay M., Poyet P., 1990. - La place de l'informatique dans les géosciences, évolution et perspectives. Géologues, n' 91 , pp. 37-49.

DETAY M., 1991. - Techniques modernes d'action sur les eaux souterraines, bilan et perspectives. Rapport Général de la Question II des XXI Journées de l'Hydraulique. Colloque de la Société Hydrotechnique de France. G.G.II.1-13.

Doussan C., 1989. - Echanges rivière-nappe, revue bibliographique. Rapport LHM/RD/89/75 du Centre d'Informatique Géologique de l'ENSMP.

Doussan C., SOMER-Portevin G., 1991. - Etude des transferts de micro-polluants de la Seine vers la nappe alluviale. Rapport d'étude LED-AFBSN-CIG-CIRSEE.

MEGNIEN C., et al., 1970. - Atlas des nappes aquifères de la région parisienne. Ed. du BRGM.

Megnien C., 1979. - Hydrogéologie du centre du bassin de Paris. Mémoire du BRGM n 98, 530 p., 270 fig.

MARLE C., 1967. - Ecoulement monophasique en milieu poreux. Revue de l'IFP, vol. XXII, $\mathrm{n}^{\bullet} 20$.

Pennequin D., Suzanne P., D'Arras D., 1991. - SOPHOS Modèle de gestion optimale de la nappe de Croissy. XXI' Journées de l'Hydraulique, SHF, Question II, Rapport n' 3, pp. III.3.1.6.

Portrinal D., Alla P., 1985. - Optimisation par modèle mathématique des investissements dans le cas de la nappe de Croissy. Wat. Supply, IWSA, Vol. 3, n², pp. 219-226.

SAFEGE, 1985. - Aubergenville : vulnérabilité de la nappe, évaluation des sites à risques. AFBSN-Lyonnaise des Eaux. Rapport d'étude n* 751101 .

SAFEGE, 1988. - Modele de gestion de la nappe de Croissy. Rapport final, Doc. 180.

SAFEGE, 1988. - Impact d'une pollution de trichloroéthane et de xylène véhiculée par la Seine sur les champs captants d'Aubergenville et de Croissy. Rapport d'étude $\mathrm{n}^{\circ} \mathrm{DP} / \mathrm{II} / \mathrm{S} 130 / 244$.

SAFEGE, 1989. - Enquête sur les sources de pollution potentielles de la nappe de Croissy. Rapport final $n^{*} \mathrm{SB} / \mathrm{S} 213$. 